\title{
Scan Performance and Reconfigurability of Agile Radiating Matrix Antenna Prototype
}

\author{
H. Abou Taam, ${ }^{1}$ G. Zakka El Nashef, ${ }^{1}$ M. Salah Toubet, ${ }^{1}$ E. Arnaud, ${ }^{1}$ \\ B. Jecko, ${ }^{1}$ T. Monediere, ${ }^{1}$ and M. Rammal ${ }^{2}$ \\ ${ }^{1}$ OSA Department, XLIM, UMR CNRS 7252, University of Limoges, 87060 Limoges Cedex, France \\ ${ }^{2}$ GRIT Department, Doctoral School of Science and Technologies, Lebanese University, Beirut, Lebanon \\ Correspondence should be addressed to H. Abou Taam; hussein.aboutaam@hotmail.com
}

Received 26 November 2014; Accepted 11 March 2015

Academic Editor: Vincenzo Galdi

Copyright (C) 2015 H. Abou Taam et al. This is an open access article distributed under the Creative Commons Attribution License, which permits unrestricted use, distribution, and reproduction in any medium, provided the original work is properly cited.

\begin{abstract}
This paper is dedicated to different experimental validations concerning a novel concept of beam forming and beam steering antenna. The working principle of the antenna is based on the equivalent radiating surface approach and inspired from an electromagnetic band gap antenna. The theoretical aspect and some numerical validations have been already published in the work of Abou Taam et al. (2014). Different electromagnetic behaviors have been demonstrated, such as low mutual coupling, and high gain preservation for high scanning angles values. In this paper, some of these electromagnetic behaviors will be proven experimentally by the means of two different feeding configurations.
\end{abstract}

\section{Introduction}

Current radiating systems still rely on array approach, for example, the patch, the dipole, and the waveguide slots array [1-3], in order to accomplish the principal agility functions: beam forming and beam steering. These types of arrays can be limited by the presence of mutual coupling, the occurrence of grating lobes, the radiating surface efficiency, and so forth $[4,5]$. Those limitations will tend to weaken the radiation performances of any radiating system. Moreover, such systems do not fulfill the future needs requirements, that is, low cost, compactness, and excellent electromagnetic performances. However, the development of a robust radiating system is feasible, but there is always a tradeoff between these requirements. Therefore, an alternative solution based on the equivalent radiating surface approach was proposed in order to overcome the aforementioned drawbacks of an antenna array system and make a compromise between the future needs requirements.

The proposed approach working principle constitutes the subject of an accepted CNRS (Centre National de la Recherche Scientifique) patent [6]. Accordingly, an intensive theoretical study and variant numerical validations have been published in [7], which compare the proposed radiating system to a classical antenna array. The theoretical performances comparison showed great performances in terms of low mutual coupling, better radiating surface efficiency, and efficient electronic beam steering for high scanning angles.

To recall, the radiating system is called "Agile Radiating Electromagnetic Band Gap Matrix Antenna." The matrix consists of a 1D, 2D, or conformal arrangement of several identical and jointed pixels. Each pixel is inspired from an electromagnetic band gap (EBG) antenna and presents a special radiating aperture which is square with uniform electric field distribution. The whole radiating surface (characterized by electric $\left(E_{s}\right)$ and magnetic $\left(H_{s}\right)$ fields distribution) yields, according to the equivalent radiating surface theory, the corresponding radiation pattern. All details can be found in [7].

In this paper, two experimental configurations, that is, beam forming network (BFN) + matrix antenna, will be described in order to prove the theoretical aspect, as in [7]. Sections 2 and 3.1 present the design and the manufacturing validation of the matrix antenna prototype. Section 3.2 is dedicated to the first experimental configuration consisting of forming and steering a Gaussian beam using a commercial power divider ( $N$ ways) and $N$ phase shifters assembled to 


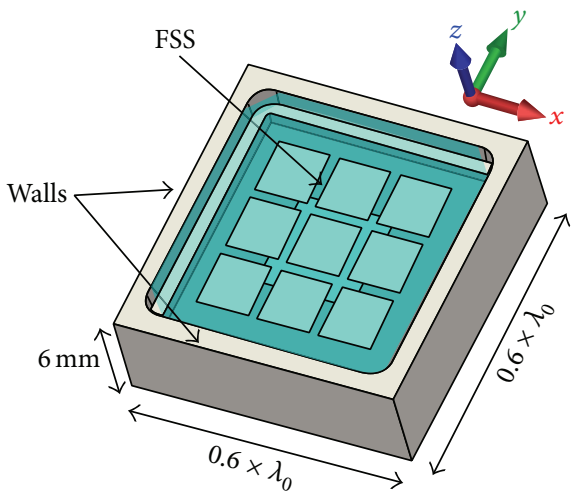

(a)

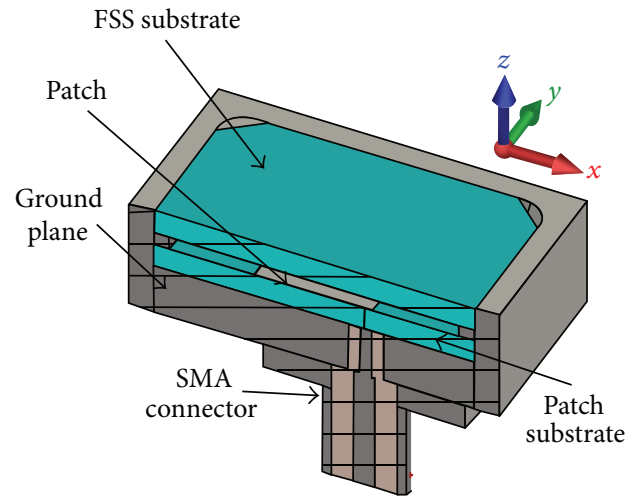

(b)

FIGURE 1: Elementary pixel: (a) perspective view and (b) inside view (cut-plane at the middle along $(0 y)$ ).

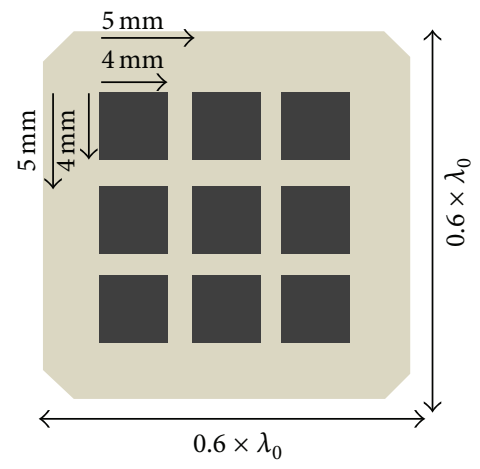

(a)

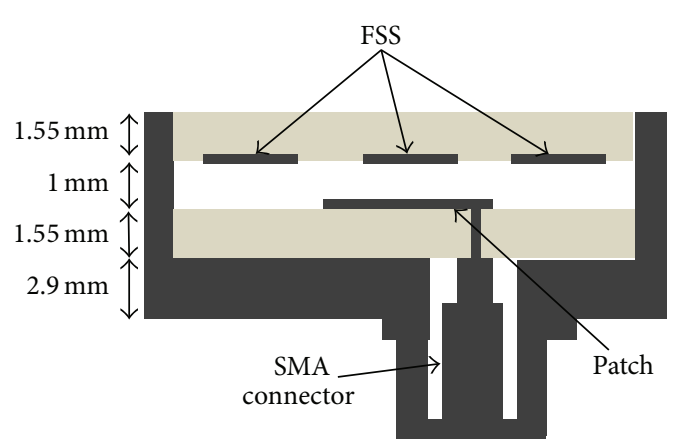

(b)

FIGURE 2: (a) FSS dimensions. (b) Detailed dimensions heights of the pixel (inside view, cut-plane at the middle along $(0 y)$ ).

the matrix antenna ( $N$ pixels). Section 3.3 describes the second configuration which is similar to the first one but with a different law of excitations (magnitudes and phases) in order to form and steer a sectorial beam. Those experimental configurations will enable us to demonstrate the accuracy of the matrix antenna and its beam forming and beam steering capabilities. Conclusions and perspectives are given in Section 4.

\section{Design and Manufactured Prototype}

The first step in designing the matrix antenna is the modeling of the EBG pixel. Figure 1 shows the EBG pixel design using full-wave simulation software (CST Microwave Studio). As described in [7], the pixel is a resonant cavity formed between a ground plane and a FSS placed above it in the $z$ direction and surrounded by four metallic walls. The FSS is composed of a centered and periodic assembly of 9 square patches $(4 \times$ $4 \mathrm{~mm}^{2}$ ) directly printed at the bottom of the FSS dielectric substrate ( $R O 4003 C$ by Rogers). Figure 2(a) shows the dimensions of the printed FSS in detail. This FSS presents a negative reflection phase value $[8,9]$ which makes the cavity height lower than $\lambda_{0} / 30$, as shown in Figure 2(b) (cavity height $=$ $1 \mathrm{~mm}, f_{0}=8.2 \mathrm{GHz}$, pixel bandwidth $\left.=5 \%\right)$. The feeding system of this pixel consists of a patch printed on a dielectric substrate $(R O 4003 C)$ and located at the EBG cavity center (Figure 2(b)). However, other feeding systems can be used such as dipoles, wave guides, and slots. It is necessary to mention that a study has been made to characterize the dielectric substrate where the obtained permittivity is equal to 3.48 and the loss tangent is equal to 0.0027 at the considered bandwidth $(8-8.4 \mathrm{GHz})$. However, one can use a different type of FSS [10] with different values of reflection coefficients and the structure principle will never change. The pixel principle is also valid for any frequency ranges.

After completing the optimization and the design of the EBG pixel, the next step is to form a $1 D$ matrix antenna which consists of 17 jointed pixels spaced by $0.6 * \lambda_{0}$, as shown in Figure 3. The structure dimensions are $\left(378 \times 30 \times 6 \mathrm{~mm}^{3}\right)$. The total height of the matrix antenna $(6 \mathrm{~mm})$ corresponds to the cavity $(1 \mathrm{~mm})$, the ground plane $(1.9 \mathrm{~mm})$, and the substrates heights $(3.1 \mathrm{~mm})$, as shown in Figure 2(b).

Figure 4 shows the manufactured prototype of the considered matrix antenna designed at $f_{0}=8.2 \mathrm{GHz}$. The matrix antenna is placed at the top of a metallic support which presents an extension along the $y$ direction $(378 \times 180 \times$ $2 \mathrm{~mm}^{3}$ ). On the bottom of the metallic support, a BFN is installed in order to feed the pixels of the matrix antenna. 


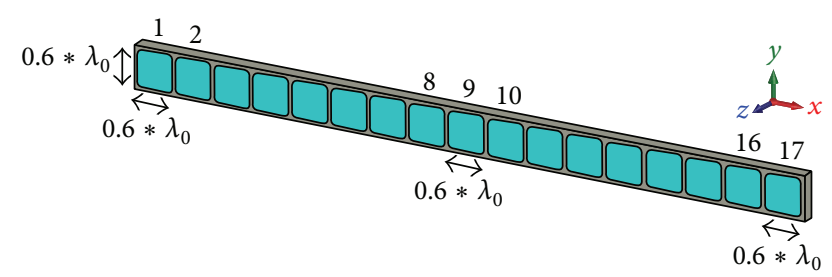

FIGURE 3: The designed prototype of $1 \mathrm{D}$ matrix antenna formed by 17 jointed pixels linearly spaced by $0.6 * \lambda_{0}$.

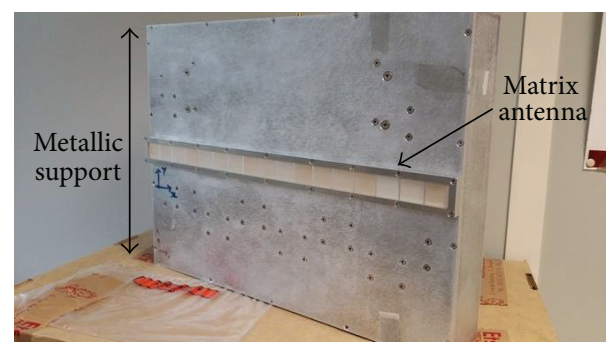

FIGURE 4: Matrix antenna manufactured prototype placed at the top of a metallic support.

More details on the BFN will be presented in Sections 3.2 and 3.3.

The next section focuses on the experimental validation of the matrix antenna leading to prove the robustness of the theoretical concept and the efficiency in beam forming and beam steering.

\section{Experimental/Numerical Validations}

3.1. Matching and Mutual Coupling Coefficients. The matrix antenna concept was validated through a numerical simulation, as described in [7]. Experimentally, a first validation of the prototype (Figure 4) was done by measuring each pixel matching coefficient in the matrix and the mutual coupling coefficient as well. Figure 5 shows the comparison between the measurement and the simulation of different pixel matching coefficients in the matrix. We can clearly see that a good agreement is obtained. The different pixels are matched to $-10 \mathrm{~dB}$ over a bandwidth of $5 \%(8-8.4 \mathrm{GHz})$. The other pixels, which were not illustrated, showed the same agreement between the measurement and the simulation, in terms of matching coefficient.

Moreover, as mentioned in [7], one of the advantages of the matrix antenna is to limit each elementary radiating aperture at the pixel's dimensions in order to not disturb the neighboring pixels [7]. Therefore, a low mutual coupling between neighboring pixels has been observed. In order to validate the low mutual coupling performances, Figure 6 shows the comparison between simulated and measured mutual couplings of the central pixel (number 9) and their adjacent ones (resp., pixels numbers 8 and 7). The obtained results show a very good agreement and the highest obtained level of mutual coupling is less than $-20 \mathrm{~dB}$.

Next in Sections 3.2 and 3.3, the experimental evaluations of matrix antenna radiation performances will be carried out.

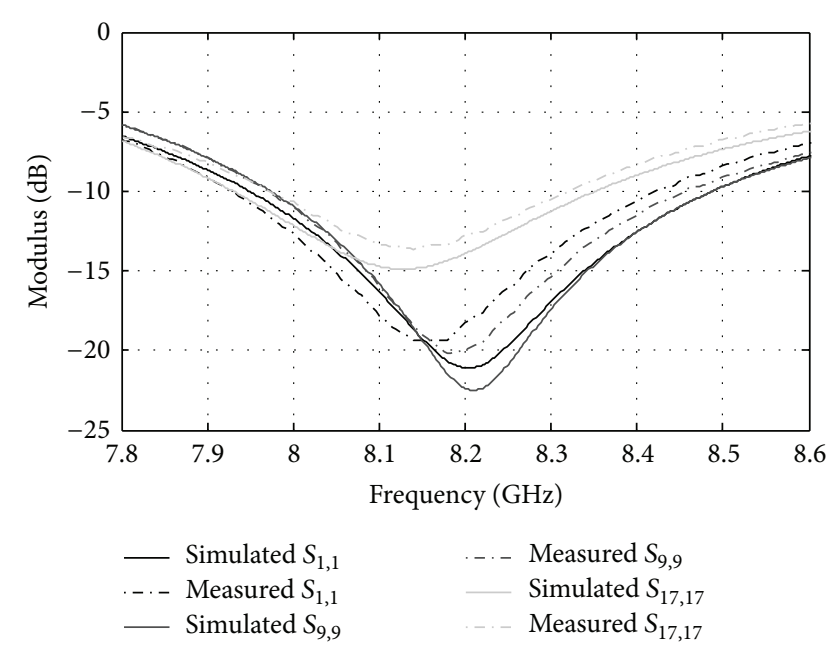

FIgURE 5: Simulated and measured matching coefficients corresponding to central and border pixels.

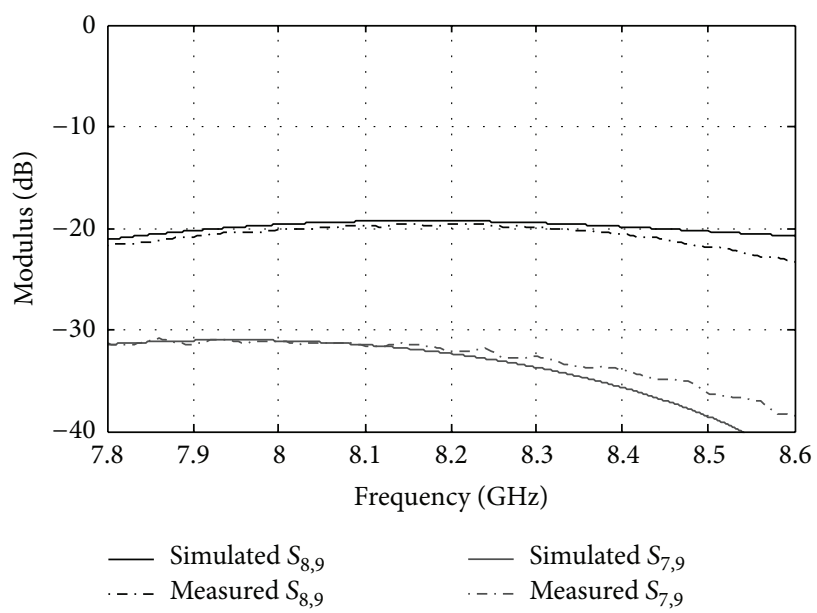

FIGURE 6: Simulated and measured coupling coefficients from the central pixel to first left neighboring one $\left(S_{8,9}\right)$ and to second left neighboring one $\left(S_{7,9}\right)$.

Two different configurations were realized to show the beam forming and beam steering capabilities of the matrix antenna. On one hand, a Gaussian type of radiation patterns will be illustrated and validated and, on the other hand, a sectorial type of radiation patterns is illustrated and validated as well.

3.2. Experimental Gaussian Beam Validation. In spite of all the undesirable radiation effects of the Gaussian beam, it is still the most common and used beam radiation type in antenna systems. This type of beam leads to a maximum gain compared to a different form of beams, that is, sectorial beam. In this paragraph, an experimental validation using a passive BFN composed of commercial equimodulus and equiphase power divider (Clear Microwave, Inc., 18-Way power divider) and 17 analog phase shifters (980-4K, Weinschel Aéroflex) will be demonstrated in order to obtain a classical Gaussian radiation beam. The low cost phase shifters are used to manually 


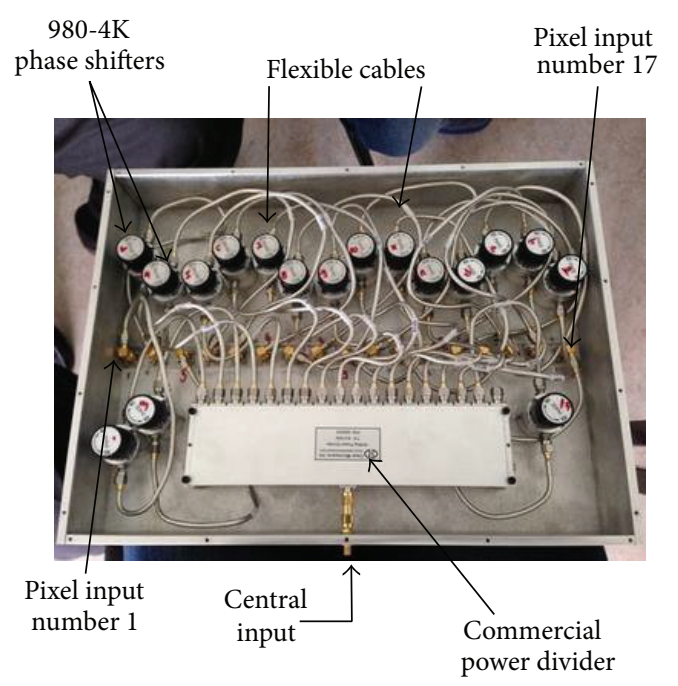

FIGURE 7: Beam forming network placed at the metallic support's bottom leading to obtain a Gaussian radiation beam.

set the steering weights. Figure 7 shows the BFN placed on the metallic support's bottom at the vicinity of the matrix antenna.

A full-wave simulation of the matrix antenna is performed with CST Microwave Studio. The matrix was associated with the measured passive BFN (touchstone file) using a cosimulation technique on CST and the whole system (measured BFN + simulated antenna) was simulated in order to compare correctly the measured and the simulated radiation patterns.

Two cases of figures are considered: the broadside direction and the steered direction at $30^{\circ}$ pointing angle. First of all, the simulated and measured matching coefficients of the global system are shown. The results show a good agreement in both cases $\left(0^{\circ}\right.$ and $\left.30^{\circ}\right)$ and the system is matched (Figure 8).

Then, Figures 9(a) and 9(b) show the comparison between measured and simulated radiation patterns for $0^{\circ}$ and $30^{\circ}$ pointing angles at $8.2 \mathrm{GHz}$. A very good agreement between different radiation patterns can be observed. The main lobes are maintained correctly leading to prove the theoretical concept. It is necessary to mention that the agreement between simulation and measurement is also obtained on the whole bandwidth $(8-8.4 \mathrm{GHz})$.

3.3. Experimental Sectorial Beam Validation. One cannot ignore the undesirable radiation effects of the Gaussian beam, for example, high side lobe levels, back radiation, and particularly $3 \mathrm{~dB}$ gain variation in the aperture. However, there are a lot of techniques which permit us to avoid these undesirable effects and even overcome them. The proposed methodology, in this paper, is to generate a special sectorial beam radiation in order to confine the electromagnetic energy between two angles. Such beam presents a quasiconstant gain over a desired angular range, as illustrated in Figure 10(b). It will also present a low side and back lobes levels. Moreover, the sectorial beam pattern overcomes the Gaussian beam pattern

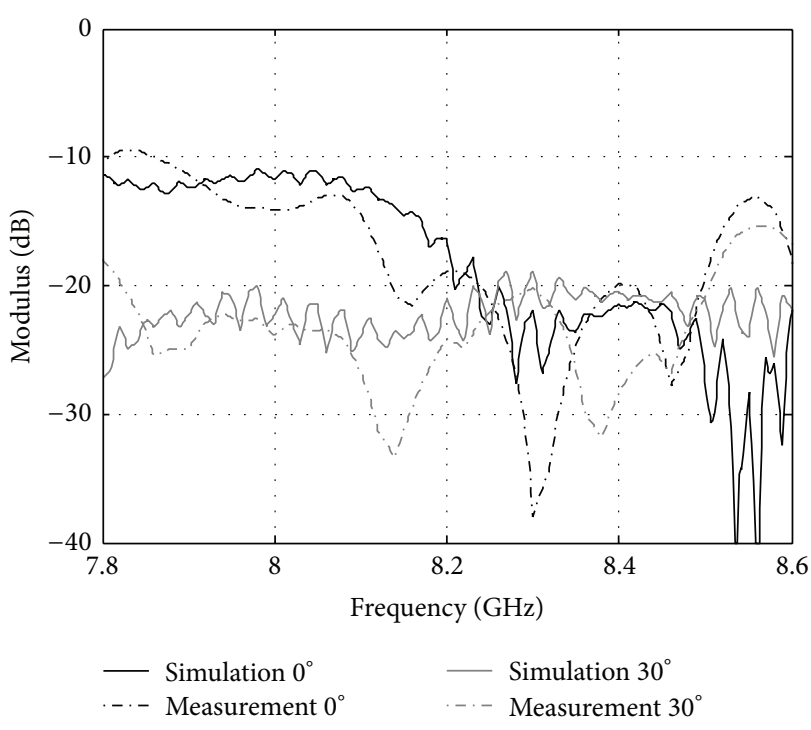

Figure 8: Simulated and measured matching coefficients of the global system for the first configuration: (a) broadside direction and (b) $30^{\circ}$ pointing angle.

(Figure 10(a)), in terms of angular range covering. In order to be explicit, Figure 11 illustrates two scenarios presenting a beam steering of Gaussian and sectorial radiations patterns in order to cover a defined angular range " $\Delta \theta^{\circ}$." The comparison demonstrates, as shown in Figure 11(a), the occurrence of radiations halls ( $-3 \mathrm{~dB}$ relative to max) when Gaussian beams are used, leading to multiplying the number of beams (dotted red shapes) in order to fill the gaps and cover the whole defined " $\Delta \theta^{\circ}$." However, the defined " $\Delta \theta^{\circ}$ " can be covered properly by using a reduced number of sectorial beams, as shown in Figure 11(b).

To recall, the radiation patterns, according to equivalent radiating surface theory, are obtained approximately by a spatial Fourier transform (FT) of the near field distribution existing on the antenna's radiating surface. For example, equimodulus and equiphase excitation law enables us to obtain a Gaussian beam which has almost the shape of a cardinal sine. Contrariwise, the proposed idea is to obtain a sectorial radiation pattern from a cardinal sine excitation. To carry out, the proposed idea consists of feeding the pixels of the matrix antenna by a cardinal sine excitation law. The cardinal sine signal is sampled by the pixel number. Therefore, each pixel is fed by special weight in modulus and in phase. In order to achieve that, a nonequimodulus and nonequiphase distribution circuit 1 input to 17 outputs, which is able to generate the cardinal sine excitation law, has been designed (Figure 12(a)) at $8.2 \mathrm{GHz}$ using momentum from Agilent Advanced System Design (ADS). The distribution circuit has been manufactured, as shown in Figure 12(b).

In order to experimentally validate the sectorial beam radiation, another configuration was realized, based on the first configuration presented in Figure 7, where the equimodulus and equiphase power divider was replaced by the one shown in Figure 12(b). The manufactured power divider will enable us to obtain a cardinal sine excitation law and 


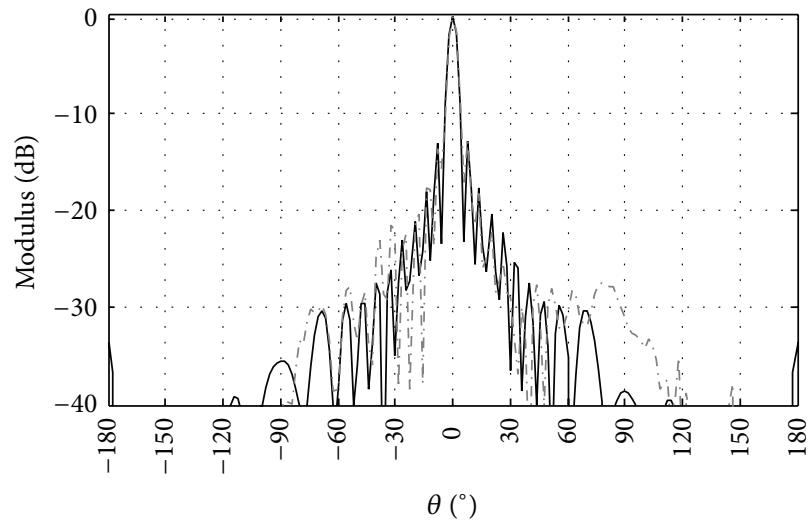

(a)

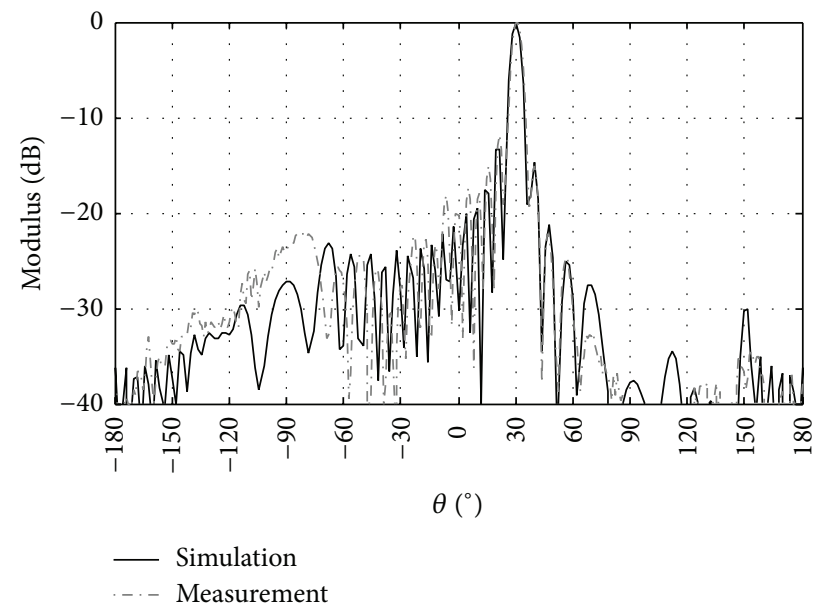

(b)

FIGURE 9: Comparison between simulated and measured normalized Gaussian beams at (a) broadside direction and (b) $30^{\circ}$ pointing angle. $f_{0}=8.2 \mathrm{GHz}$.

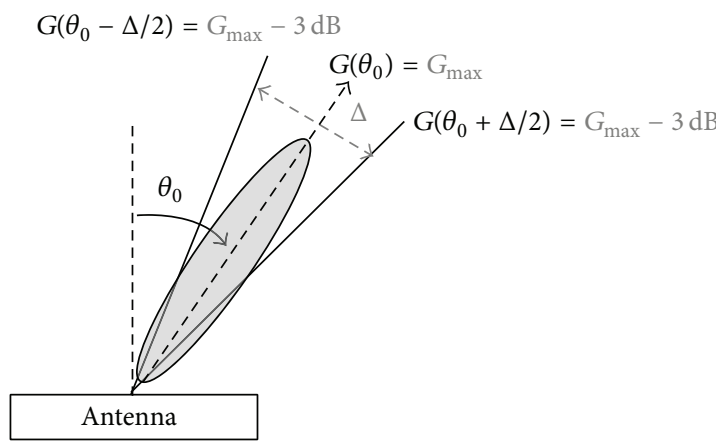

(a)

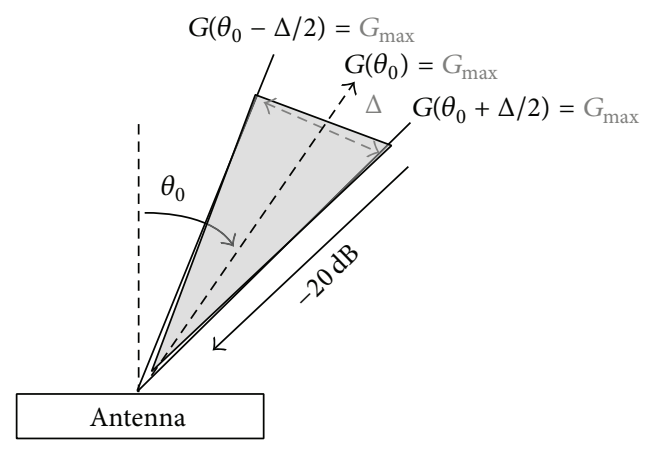

(b)

FIGURE 10: Illustration of the Gaussian beam radiation (a) and the proposed sectorial beam radiation (b) steered at $\theta_{0}$ direction.

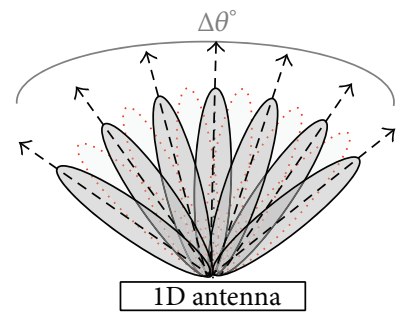

(a)

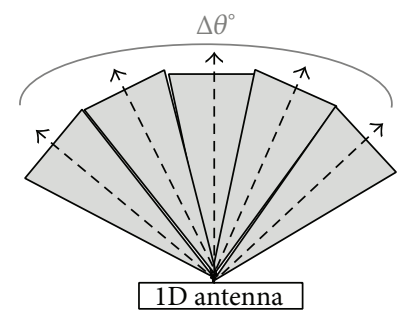

(b)

FIGURE 11: Illustration of (a) Gaussian beams and (b) sectorial beams for beam steering scenario.

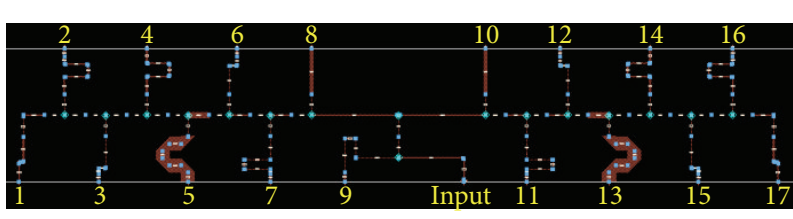

(a)

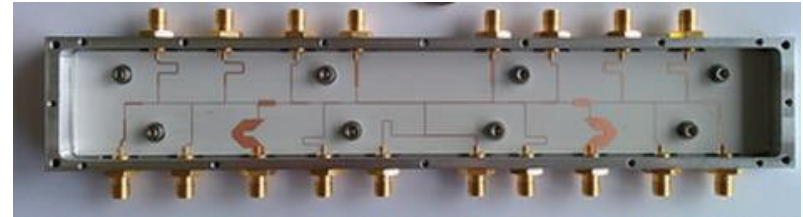

(b)

FIGURE 12: Cardinal sine power divider: (a) momentum layout and (b) manufactured prototype. 


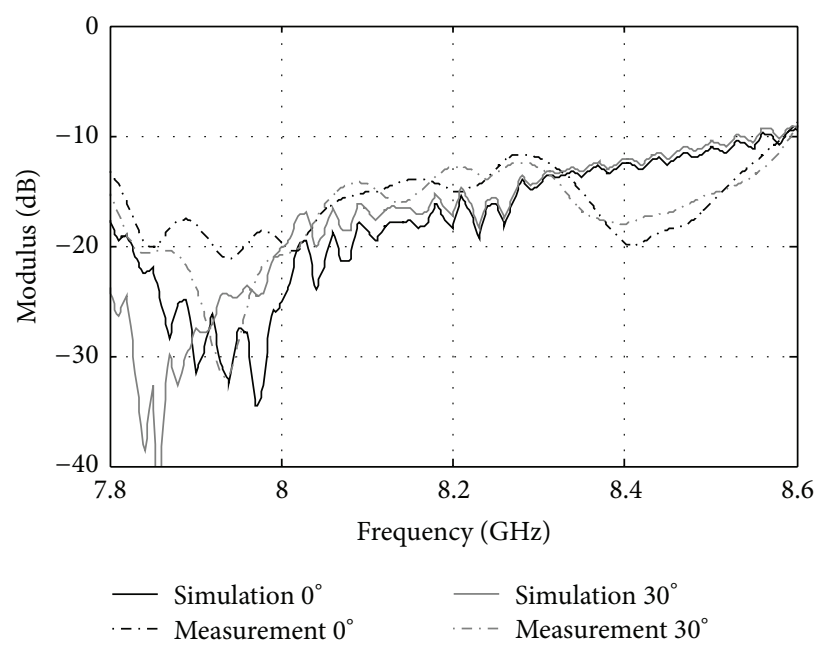

FIGURE 13: Simulated and measured matching coefficients of the global system for the second configuration: (a) broadside direction and (b) $30^{\circ}$ pointing angle.

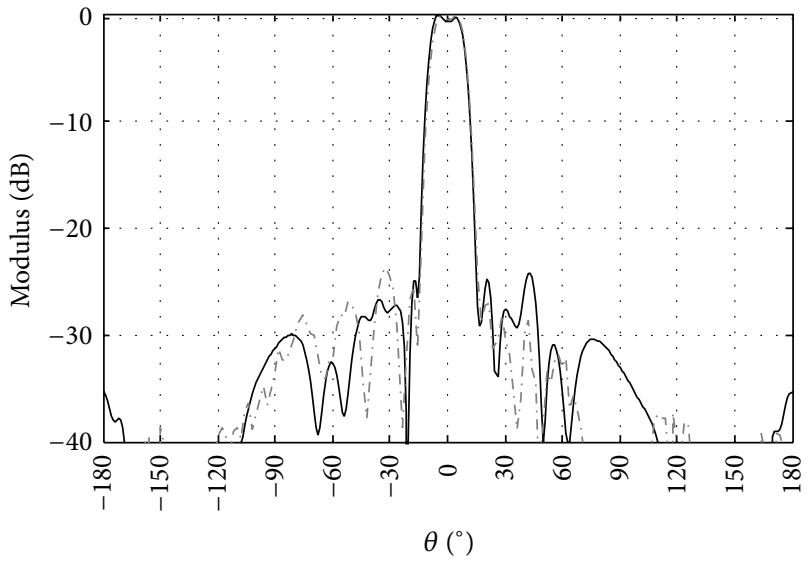

(a)

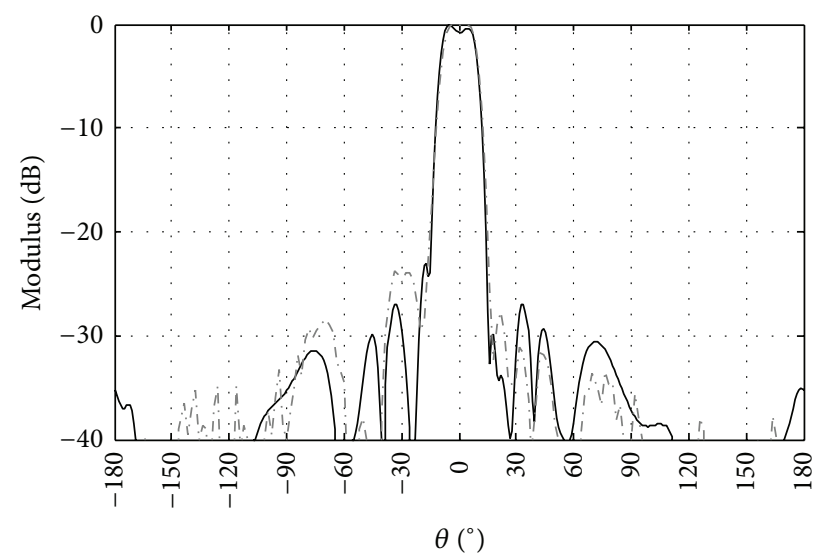

(b)

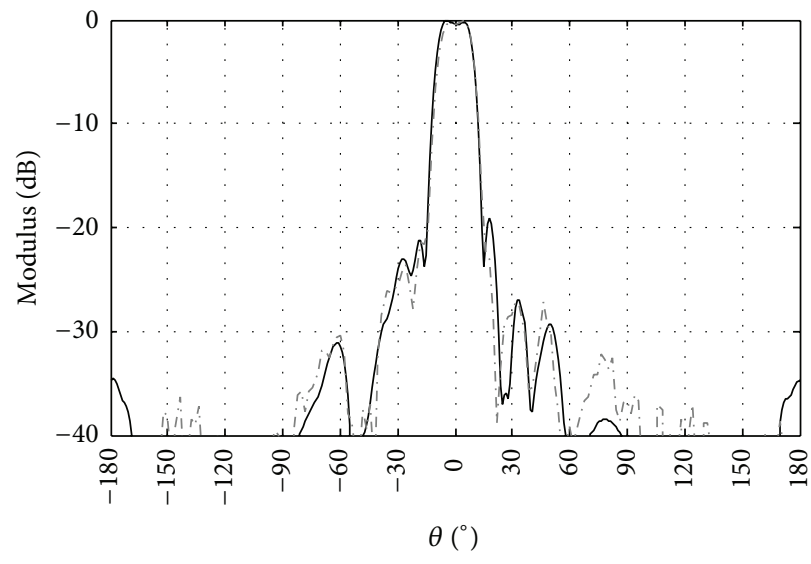

- Simulation

.... Measurement

(c)

Figure 14: Comparison between simulated and measured normalized sectorial radiation patterns at broadside direction: (a) $8 \mathrm{GHz}$, (b) $8.2 \mathrm{GHz}$, and (c) $8.4 \mathrm{GHz}$. 


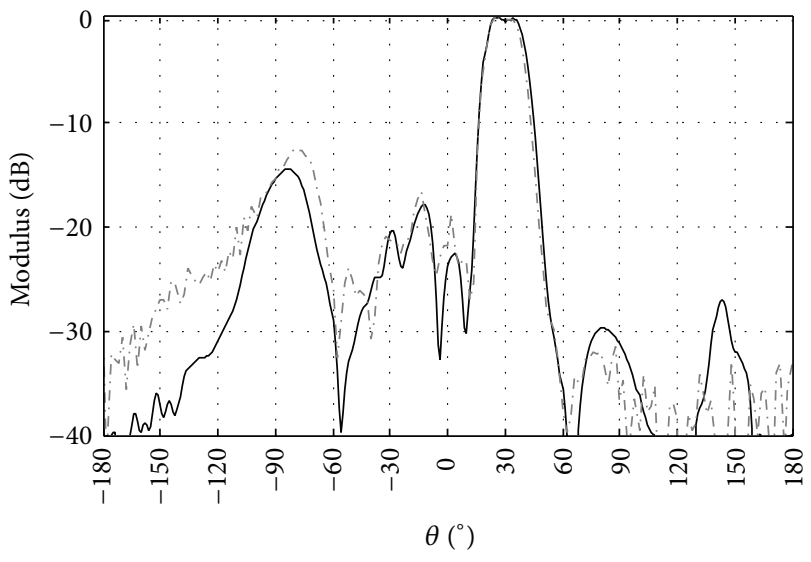

(a)

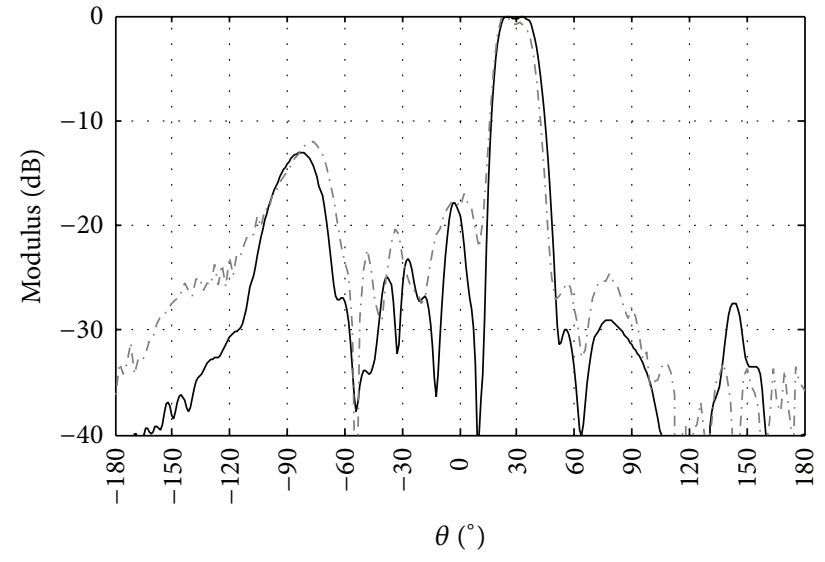

(b)

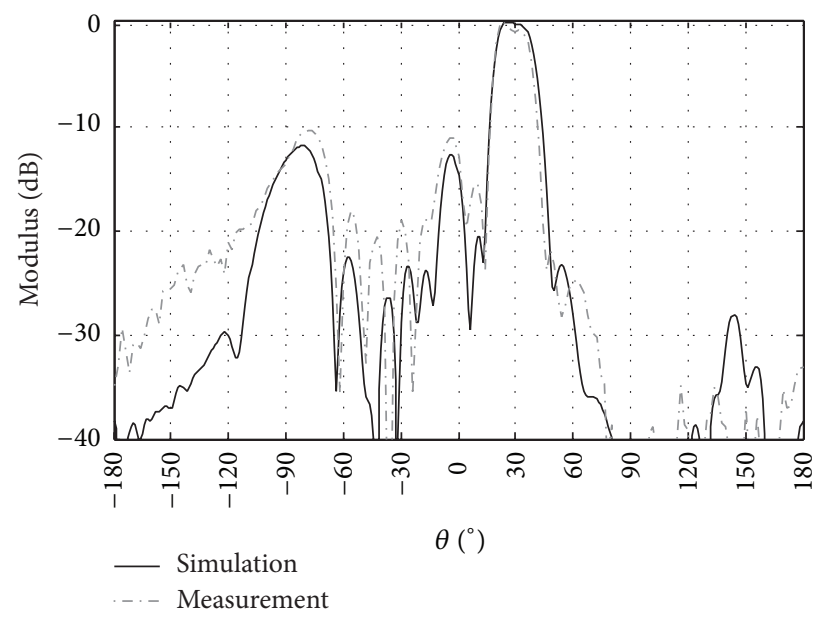

(c)

FIGURE 15: Comparison between simulated and measured normalized sectorial radiation patterns at $30^{\circ}$ pointing angle direction: (a) $8 \mathrm{GHz}$, (b) $8.2 \mathrm{GHz}$, and (c) $8.4 \mathrm{GHz}$.

the phase shifters are used to manually set the steering weights. The global system (matrix antenna + BFN) will be able to accomplish a beam forming features (i.e., by the means of the special distribution circuit) and beam steering (i.e., by the means of phase shifters).

The simulated and the measured matching coefficients on the central input for two cases $\left(0^{\circ}\right.$ and $\left.30^{\circ}\right)$ are shown in Figure 13. The results show that the final prototype is matched over the whole bandwidth $(8-8.4 \mathrm{GHz})$. Figures 14 and 15 show the comparison between simulated and measured radiation patterns, respectively, for $0^{\circ}$ and $30^{\circ}$ pointing angles. A good agreement is obtained and both radiation patterns have a sectorial shape with a quasiconstant gain over an angular range of $18^{\circ}$. The side lobe and the back radiation levels are, respectively, lower than $-22 \mathrm{~dB}$ and $-35 \mathrm{~dB}$, for broadside direction over 8-8.4 GHz. In the steered direction, the main lobe is maintained correctly and the observed side lobes level stay less than $-12 \mathrm{~dB}$ for the worst case (Figure 15(c); $f=8.4 \mathrm{GHz}$ ). The sectorial beams specifications are correctly maintained at the desired bandwidth. Moreover, a rise in grating lobe levels is observed in the steered direction (i.e., $\left.30^{\circ}\right)$ due to the spacing between pixels $\left(0.6 \times \lambda_{0}\right)$.

\section{Conclusion}

This paper was dedicated to demonstrate experimentally the beam forming and beam steering capabilities of an innovative antenna called "Agile Radiating Electromagnetic Band Gap Matrix Antenna." The antenna theoretical concept was already published in [7]. In this paper, the design of an elementary pixel and the 1D EBG matrix (17 pixels) were described. The proposed 1D matrix antenna was manufactured and was experimentally validated using two different configurations: one that generates a classical Gaussian beam and the other generates a sectorial beam. The experimental results showed that the matrix antenna presents a low level of mutual coupling and high efficiency in terms of radiating performances. In addition, the matrix antenna is a polyvalent antenna where it can generate any type of radiation at any desired frequency by only a simple control of the magnitude 
and the phase making it a robust solution for future radar or telecommunication applications.

Many short- and long-term perspectives are being investigated. The most recent one is the reduction of the grating lobes effect which was already demonstrated theoretically in [11]. An experimental prototype will be manufactured to validate the theoretical concept.

Finally, the matrix antenna provides a robust, reliable, and efficient solution to new design capabilities for agile antenna applications where the beam forming and beam steering are sought criteria.

\section{Conflict of Interests}

The authors declare that there is no conflict of interests regarding the publication of this paper.

\section{References}

[1] R. J. Pogorzelski, R. P. Scaramastra, J. Huang, R. J. Beckon, S. M. Petree, and C. M. Chavez, "A seven-element s-band coupledoscillator controlled agile-beam phased array," IEEE Transactions on Microwave Theory and Techniques, vol. 48, no. 8, pp. 1375-1384, 2000.

[2] E. A. Monastyrev, S. L. Kevruh, V. A. Moloshnikov, P. E. Denisov, A. V. Akimov, and A. A. Ponomarev, "LTCC based planar modules for X-band aesa," in Proceedings of the 21th International Crimean Conference on Microwave and Telecommunication Technology (CriMiCo '11), vol. , no, pp. 195-197, September 2011.

[3] M. Ettorre and R. Sauleau, "Reconfigurable multi-beam pillbox antenna for millimeter wave automotive radars," in Proceedings of the 9th International Conference on Intelligent Transport Systems Telecommunications (ITST '09), pp. 87-90, October 2009.

[4] S. D. Gupta, "Effect of mutual coupling in $\mathrm{E}$ and $\mathrm{H}$ plane on microstrip antenna array conformal on cylindrical surface," in Proceedings of the International Conference on Signal Processing and Communication (ICSC '13), pp. 149-154, December 2013.

[5] F. Kitson and L. Griffiths, "Grating lobe effects in linear adaptive receiving arrays," in Proceedings of the International IEEE Antennas and Propagation Society International Symposium, vol. 18, pp. 379-382, June 1980.

[6] B. Jecko, M. Hajj, R. Chantalat, and M. S. Toubet, "Antenne élémentaire et antenne réseau mono ou bidimensionnelle correspondante," PCT Patent PCT/EP2012/076509, Université de Limoges, Limoges, France, 2013.

[7] H. Abou Taam, M. Salah Toubet, T. Monediere, B. Jecko, and M. Rammal, "A new agile radiating system called electromagnetic band gap matrix antenna," International Journal of Antennas and Propagation, vol. 2014, Article ID 342518, 7 pages, 2014.

[8] D. Sievenpiper, L. Zhang, R. F. J. Broas, N. G. Alexöpolous, and E. Yablonovitch, "High-impedance electromagnetic surfaces with a forbidden frequency band," IEEE Transactions on Microwave Theory and Techniques, vol. 47, no. 11, pp. 2059-2074, 1999.

[9] R. Chantalat, L. Moustafa, M. Thevenot, T. Monediere, and B. Jecko, "Low profile EBG resonator antennas," International Journal of Antennas and Propagation, vol. 2009, Article ID 394801, 7 pages, 2009.

[10] Y. Ge, K. P. Esselle, and T. S. Bird, "The use of simple thin partially reflective surfaces with positive reflection phase gradients to design wideband, low-profile EBG resonator antennas," IEEE
Transactions on Antennas and Propagation, vol. 60, no. 2, pp. 743-750, 2012.

[11] H. A. Taam, M. S. Toubet, T. Monediere, B. Jecko, and M. Rammal, "Interests of a 1D EBG Matrix compared to a patch array in terms of mutual coupling and grating lobe," in Proceedings of the 7th European Conference on Antennas and Propagation (EuCAP '13), pp. 1045-1048, April 2013. 

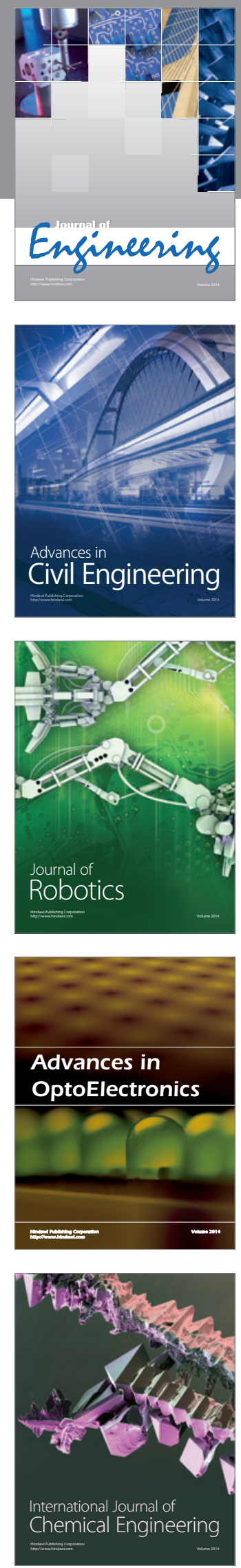

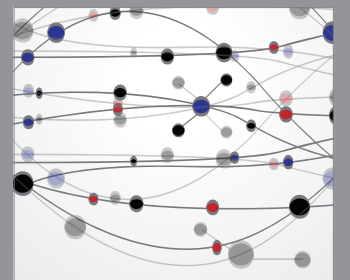

The Scientific World Journal
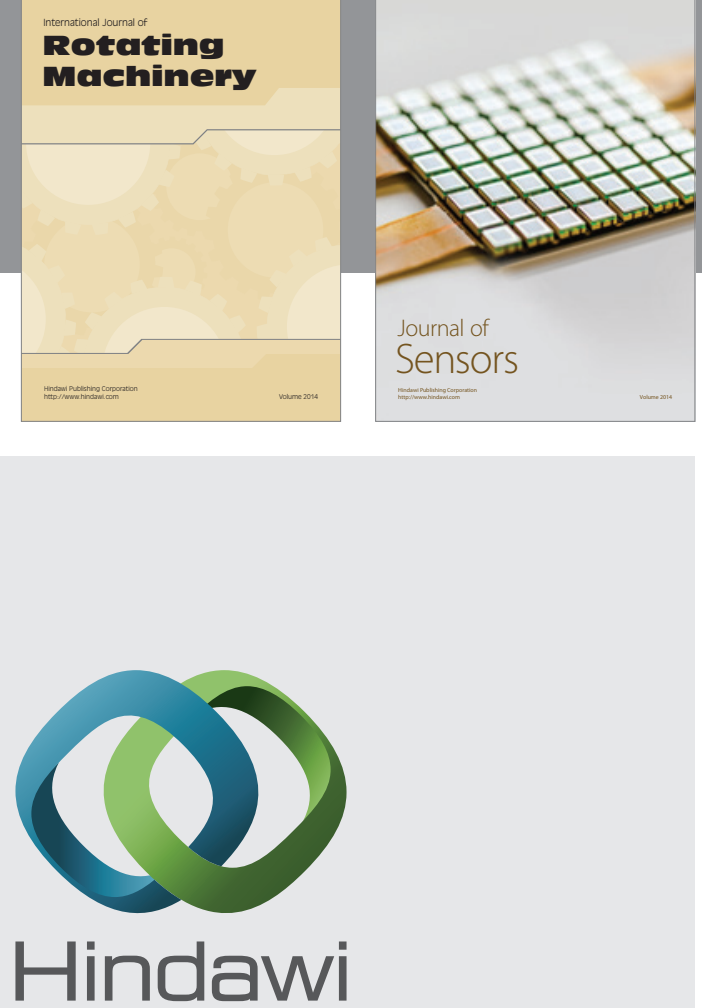

Submit your manuscripts at http://www.hindawi.com
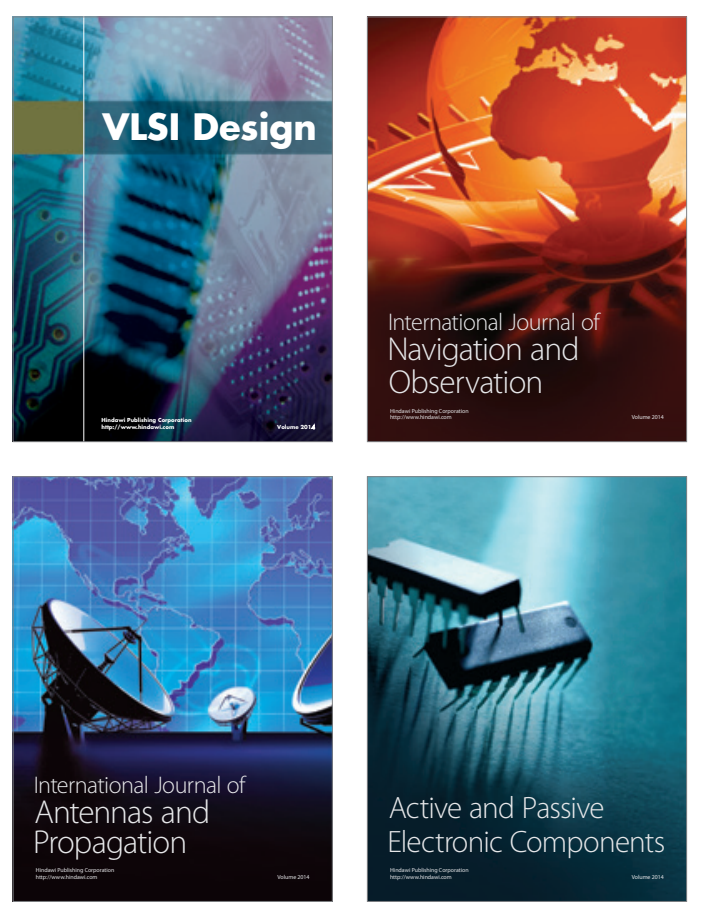
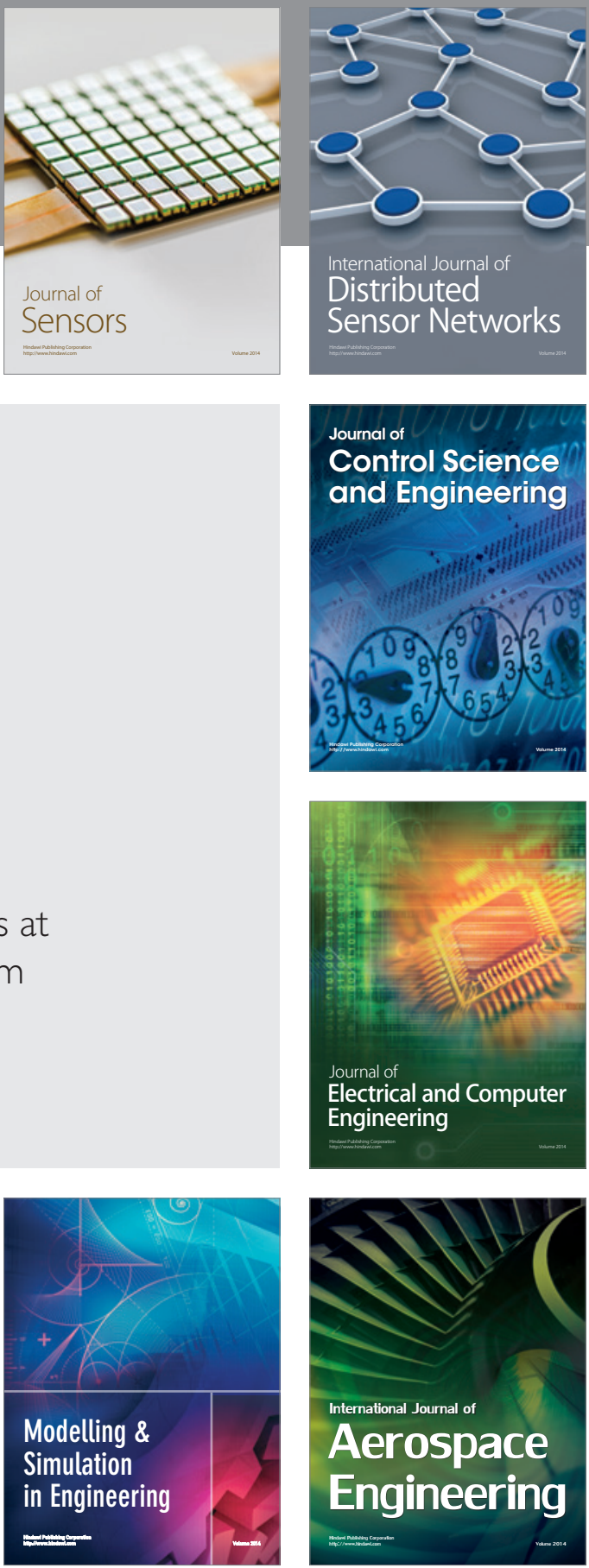

Journal of

Control Science

and Engineering
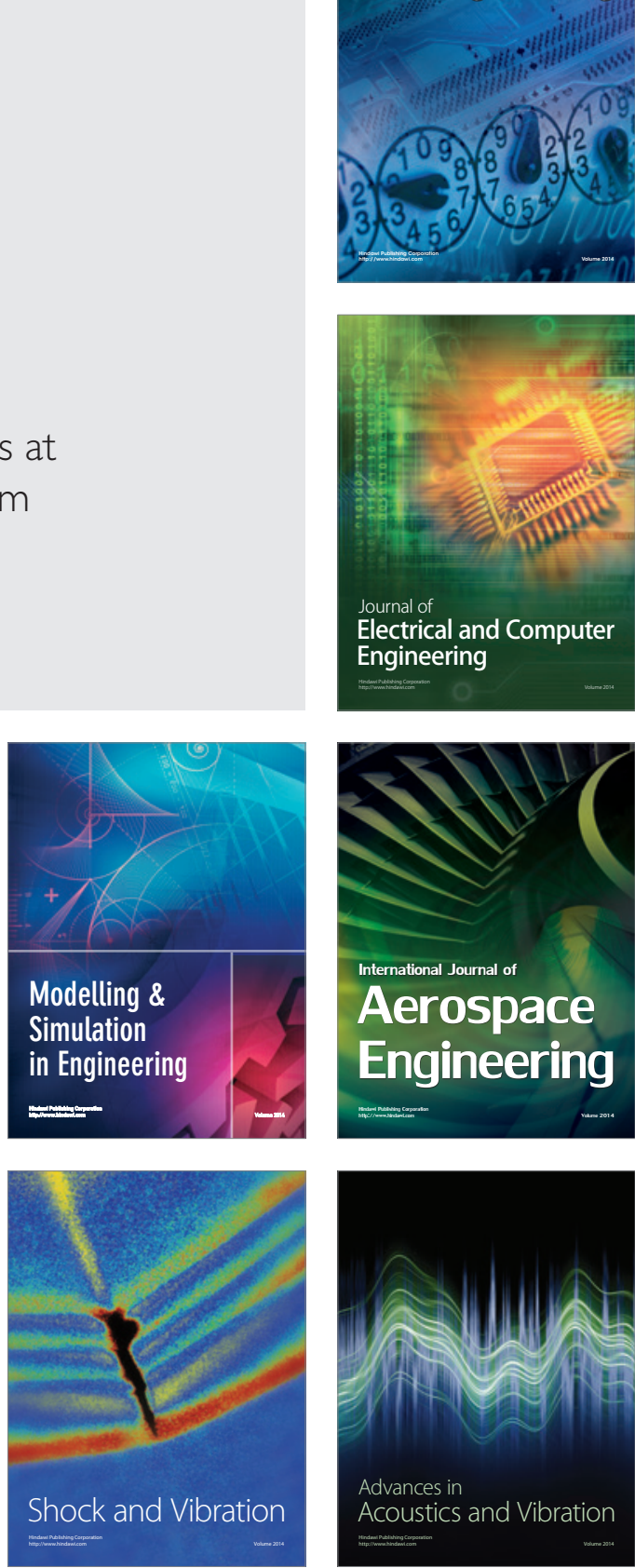